\title{
Saint-Simon et Auguste Comte : la fin d'une collaboration, 1822-1824.
}

\author{
Michel Bourdeau
}

\begin{abstract}
A Mary Pickering, dont la monumentale biographie de Comte rend et rendra encore longtemps d'immenses services.
\end{abstract}

« Il y a donc un problème historique du positivisme, puisqu'il est impossible de ne pas se demander quelle fut la part de Saint-Simon dans sa formation. Ce problème, Comte le connaissait si bien qu'il l'a nié jusqu'à la fin de sa vie ». Henri Gouhier

Résumé : La question des rapports entre Comte et Saint-Simon est toujours en suspens, la version qu'en a donnée le premier étant très sujette à caution. Pour tenter d'y voir clair, il convient de commencer par régler certaines questions factuelles. Les pages qui suivent s'en tiennent aux années qui séparent les publications, dans des périodiques édités par Saint-Simon, du Plan des travaux scientifiques nécessaires pour réorganiser la société (mai 1822-mai 1824) et qui s'achèvent sur une rupture. Grâce à la récente édition des CEuvres complètes de Saint-Simon, il est possible de dégager ce que ses écrits de cette période nous apprennent sur les relations entre les deux hommes.

Mots-clés: Auguste Comte, Saint-Simon, politique positive, philosophie de l'histoire, industrialisme, Condorcet.

Summary : The question of the relationship between Comte and Saint-Simon is still a very controversial issue: Comte, who for seven years acted as secretary to Saint-Simon, has been afterwards very unfair to him. The first step toward a clarification is to settle some facts. The paper focuses on what happened between the two publications of the Plan des travaux scientifiques nécessaires pour réorganiser la société (May 1822- May 1824), a crucial episode which ended with Comte breaking off definitively with Saint-Simon. Thanks to the new edition of the Collected Works of Saint-Simon, it is possible to find, in what was published at that time, an account of the way Saint-Simon understood his collaboration with his secretary.

Keywords : Auguste Comte, Saint-Simon, Industrialism, positive policy, philosophy of history, Condorcet

La question des rapports de Comte et de Saint-Simon est connue pour être controversée et risque de le rester encore pour un bon moment. Comte fut secrétaire de Saint-Simon de 1817 à 1824. Un temps sous le charme du " père Simon ", Comte ne tarda pas à se brouiller avec lui et en vint à nier toute dette envers celui où il ne voulait plus voir qu'un "jongleur dépravé ». Durkheim, pour minimiser sa dette envers l'auteur du Cours, ne voulait plus voir en lui qu'un saint-simonien et Hayek, pour de tout autres raisons, se rangeait également à cette avis. Les travaux de Gouhier, qui s'inscrivent dans le cadre des débats entre philosophie et sociologie dans l'université française de l'entre-deux-guerres, avaient pour but de réfuter Durkheim. Plus récemment, dans sa magistrale biographie, Mary Pickering a repris la question sur de nouveaux frais pour arriver à une conclusion plus nuancée, soulignant la dette de Comte envers celui qui fut un temps son mentor, mais montrant également qu'il y avait de bonnes raisons d'attribuer à Comte un certain nombre de textes, ou d'extraits de textes, que Saint-Simon a publié sous son nom et qui continuent à être lus comme tels. Plus récemment enfin, les éditeurs des Euvres complètes ont choisi d'ignorer les conclusions de Pickering et de minimiser la contribution de Comte ${ }^{1}$.

Cette édition n'en rend pas moins un service inappréciable en ce que, pour la première fois, elle rend facilement accessible l'ensemble du dossier, d'une composition on ne peut plus complexe. Ceci vaut plus particulièrement de la période à laquelle je m'en tiendrai, celle qui encadre les deux éditions du premier Système de politique

\footnotetext{
${ }^{1}$ Henri Saint-Simon, Euvres complètes, éditées par Juliette Grange, Pierre Musso, Philippe Régnier et Frank Yonnet ; Paris, PUF, 2012, 4 vol., 3444p. ; désormais abrégé en $O C$ suivi du numéro du volume.
} 
positive, le Plan des travaux scientifiques nécessaires pour réorganiser la société, que Comte avait l'habitude de désigner comme son opuscule fondamental: la première, dans la troisième partie du Système industriel (avril-mai 1822); la seconde, dans le troisième cahier du Catéchisme des industriels (avril 1824). Ces deux années correspondent à un moment crucial où les relations des deux hommes se détériorent pour finir par une rupture. Saint-Simon meurt un plus tard le 19 mai 1825. De cette rupture, nous ne possédons qu'un seul récit, celui de Comte, que l'on est en droit de tenir pour sujet à caution. Bien plus, sur cette période, la correspondance de Comte est étrangement muette : entre septembre 1820 et avril 1824, nous ne possédons aucune lettre ${ }^{2}$, alors qu'elle reprend, abondante, aussitôt la rupture consommée, — de sorte qu'il s'agit pour une bonne part d'un récit rétrospectif.

Pour la période concernée, les textes de Saint-Simon forment un ensemble de quelque deux cents pages, comprenant un certain nombre d'inédits ou de quasi inédits. Ils nous permettent de suivre dans les détails l'évolution de l'auteur, de repérer les références ou allusions à Comte et de les confronter à la version que nous en donne celui-ci. Grâce à eux, il est possible, et c'est l'objet du présent article, de revisiter la question toujours ouverte des rapports de Comte et de Saint-Simon, plus précisément de leur rupture, ce qui sera fait en trois temps. Après avoir rappelé où en était Saint-Simon en 1822, et le programme qu'il s'était fixé, on examinera ce que ces textes nous apprennent sur la façon dont il concevait la collaboration avec son secrétaire. Une dernière partie sera consacrée aux circonstances de la rupture et aux enseignements que l'on peut en tirer.

\section{Saint-Simon}

Quelques rappels. Commençons par rappeler où en est Saint-Simon au début de la période qui nous occupe, à savoir au printemps 1822. Dans son évolution, la chute de l'Empire a marqué une profonde césure. La promulgation de la Charte constitutionnelle apportait une grande bouffée d'air frais. A la chape de plomb idéologique imposée par Napoléon succédait une liberté d'expression, toute relative il est vrai, mais qui permit la prolifération de brochures, pamphlets, périodiques, prospectus de toute sorte, dont la production saintsimonienne n'est qu'un échantillon. Ce changement dans les conditions sociales de la vie intellectuelle s'est accompagné chez Saint-Simon d'une nette réorientation de son programme. Tout au long de sa carrière intellectuelle, celui-ci a poursuivi deux buts : l'un, pratique, terminer la révolution, réformer la société ; l'autre théorique, fonder une science de l'homme et de la société. Après 1815, la part respective attribuée à chacun de ses deux aspects se modifie. Le projet encyclopédique passe au second plan, au profit de la dimension proprement politique ${ }^{3}$. L'auteur du Mémoire sur la science de l'homme se lance dans la mêlée. Il multiplie les adresses au Roi, aux membres de la chambre des députés, aux électeurs, et prend position en faveur du parti industriel et de ce qu'il en viendra à appeler l'industrialisme.

Si Saint-Simon ne s'écartera pas de la ligne ainsi fixée, une évolution n'en est pas moins manifeste, scandée par la succession des publications, qui introduit une discontinuité en partie artificielle. C'est ainsi que paraissent et disparaissent tour à tour : 1) L'Industrie, (1816-1818) ; 2) Le Politique, (1819) ; 3) L'Organisateur, (1819$1820)$; 4) Du système industriel (1820-1822; celui qui dure le plus longtemps); 5) le Catéchisme des industriels (1823-24 ; faute d'argent, les publications s'interrompent de juin 1822 à décembre 1823); 6) Les Opinions littéraires, philosophiques et industrielles (1825). La rapide succession de ces périodiques ou semipériodiques s'explique par le climat politique de l'époque (sur la situation politique autour de 1818, voir v. g. OC III, p. 1816-17). La liberté de la presse restait très encadrée et Saint-Simon dépendait étroitement de ses commanditaires, les industriels dont il était le porte-parole ${ }^{4}$. Or ceux-ci s'effrayaient parfois de la hardiesse des positions prises par l'ancien aristocrate. C'est ainsi que, suite aux propos sur la morale terrestre publiés dans l'Industrie, certains des souscripteurs s'empressèrent d'écrire au ministre de la Police pour s'en désolidariser, ce qui entraîna rapidement la disparition du périodique (0C II, p. 1434).

Du système industriel, où est publié la première édition de l'opuscule fondamental, marque une étape importante dans l'évolution de Saint-Simon, notamment en ce qu'il s'y sépare des libéraux, dont il était très

\footnotetext{
${ }^{2} \mathrm{~A}$ une exception toutefois : la lettre à Blainville, datée du 11 avril 1825 dans la Correspondance Générale éditée par Paulo Carneiro et alii (8 vols. ; Paris: École Pratique des Hautes Etudes; La Haye: Mouton, 19731990, (désormais abrégé en $C G$ ), t. 1, p. 161), est en réalité antérieure de deux ans, puisqu'elle fait suite à la tentative de suicide de Saint-Simon, survenue le 9 mars 1823 ; voir Mary Pickering, Auguste Comte: An Intellectual Biography, vol. 1, Cambridge: Cambridge University Press, 1993, p. 231 et déjà Henri Gouhier, «Blainville et Auguste Comte », Revue d'histoire des sciences, 32-1, janvier 79, p. 59.

${ }^{3}$ Les éditeurs parlent ainsi d'« un va et vient permanent entre les grandes réflexions de science politique et la "petite" politique, conjecturelle et quotidienne » (OC IV, p. 3299).

${ }^{4}$ Il existe deux listes de souscripteurs : la première publiée par Saint-Simon lui-même en 1817 (OC II, p. 1454), la seconde par Alfred Pereire en 1906, qui se rapporte plus vraisemblablement au Système industriel (OC III 2702-2707 ; voir aussi Pierre Musso, La religion du monde industriel : analyse de la pensée de Saint-Simon, Paris, Éd. de l'Aube, 2006, p. 321 n. 44 et Henri Gouhier, La jeunesse d'Auguste Comte, Paris, Vrin, t. 3 (2 ${ }^{\mathrm{e}}$ éd.), 1970, p. 218.
} 
proche depuis le début de la restauration. Avec L'Organisateur, il avait réussi un gros « coup » médiatique. En deux mois (novembre-décembre 1819) la première livraison, qui contenait ce qu'il est convenu d'appeler la parabole de Saint-Simon, avait connu pas moins de trois éditions. Mais ce succès avait un revers. L'ouvrage fut saisi et l'auteur trainé en justice, pour un procès dont il sortit acquitté. L'Organisateur avait vécu ; mais la publicité qu'il devait au gouvernement lui valut quelques nouveaux subsides, qui lui permirent de lancer $D u$ système industriel, mais qui furent vite épuisés. C'est ainsi que les publications cessent en juin 1822 et que commence pour Saint-Simon une détresse financière analogue à celle qu'il avait connue en 1805-1806, Ternaux étant le seul industriel à lui conserver sa confiance.

Les difficultés. Avant d'entrer dans l'examen des textes, il convient de dire quelques mots des difficultés qu'ils opposent au lecteur. Les unes sont générales et tiennent au style, souvent déroutant, de Saint-Simon ${ }^{5}$; les autres sont propres aux écrits de cette période et tiennent au rôle que Comte peut avoir joué dans leur production.

«Série désordonnée de tracts et de brochures $»^{6}$, " éléments disparates [..] sans plan apparent »": très vite, le lecteur ne peut manquer d'être frappé par le caractère d'ordinaire extrêmement décousu, dispersé, des écrits de Saint-Simon, surtout après 1815, mais déjà avant. Il faut donc être tout particulièrement reconnaissant aux éditeurs des Oeuvres complètes du soin qu'ils ont pris à mettre de l'ordre dans ce qui se présente tout d'abord comme un inextricable maquis. Ce travail de critique textuelle une fois accompli, reste ensuite à comprendre ce qui en résulte. On se trouve alors face à un désordre d'une autre nature, qu'on ne saurait attribuer aux seules conditions très particulières dans lesquelles écrivait Saint-Simon : au jour le jour, obligé de suivre l'actualité et soumis à toute sorte de pressions. L'originalité de Saint-Simon, c'est d'être « un auteur peu soucieux de devenir auteur et de faire oeuvre» (OC I, p. 40), peu soucieux notamment de faciliter le travail de son lecteur. Ses négligences de composition frisent parfois l'absence de composition. Ecrivant au fil de la plume, il n'est pas rare que Saint-Simon annonce un plan pour aussitôt après l'oublier (OC II, p. $1746 \mathrm{n}$. 9). Autre procédé récurrent, le recyclage, qu'il s'agisse de textes déjà publiés ${ }^{8}$, dans l'idée sans doute que le lecteur pressé n'y verra que du feu, ou du réemploi de textes refusés, en l'occurrence un texte d'Auguste Comte $^{9}$. Si la multiplication d'annonces à quoi rien ne correspond dans la suite, de prospectus d'ouvrages qui ne verront jamais le jour ne constitue pas un mode de composition à proprement parler, elle n'en est pas moins caractéristique du style de Saint-Simon et, dès avant 1815, il écrit volontiers au futur. Certes, le fait tient bien souvent à l'arrêt inopiné d'une publication ; mais la question subsiste, à laquelle il est parfois bien malaisé de répondre : à quoi ces annonces non tenues peuvent-elles faire allusion?

Ces obstacles une fois surmontés, il en surgit un nouveau. Non seulement la pensée de Saint-Simon recèle de profondes ambiguités, qui font qu'elle a pu inspirer aussi bien des socialistes que des libéraux ${ }^{10}$; elle oscille entre différentes directions, sans bien réussir à se fixer. S'il est vrai par exemple que «Du système industriel marque le glissement du travail de philosophie politique [...] à une préoccupation plus directement pratique et idéologique $»^{11}$, on comprend alors mal comment il peut déclarer: "Tous mes travaux ultérieurs auront désormais pour objet de vous [...] faire sentir le véritable caractère de la doctrine qui doit servir de base au régime industriel» ${ }^{12}$. Pour expliquer cette situation, Mary Pickering a émis l'hypothèse que, pour $D u$ système industriel tout au moins, elle pourrait résulter du mode de composition de l'ouvrage. Tout le monde s'accorde en effet à admettre qu'il a été écrit «à quatre mains », quitte ensuite à diverger quant à ce qu'il convient d'attribuer à Saint-Simon et à son secrétaire ${ }^{13}$. Pour qui est un tant soit peu familier avec le Plan de 1822, de

\footnotetext{
${ }^{5}$ Sur ce que le style de Saint-Simon peut avoir de déroutant, voir encore Musso op.cit. p. 44-45 et Christophe Prochasson, Saint-Simon ou l'anti-Marx, Paris, Perrin, 2005, p. 62 et 72.

${ }^{6}$ Jean Dautry, Saint-Simon, Textes choisis, Paris, Editions sociales, 1951, p. 34, à propos du Système industriel.

${ }^{7} O C$ IV, p. 2755 ; les éditeurs parlent encore de «ce désordre et ce morcellement gênants, il faut bien le reconnaitre, pour la lecture en livre d'un 'système' » (OC IV, p. 3303).

${ }^{8}$ Voir par exemple $O C$ IV, p. $2762-63$ (Troisième partie du Système industriel) qui reprend un fragment de Des Bourbon et des Stuart (OC IV, p. 2741-42), ou encore OC IV, p. 3068-71 (Opinions littéraires, philosophiques et industrielles) qui reprend un fragment du Catéchisme des industriels (OC IV, p. 2979-82).

${ }^{9} O C$ III, p. 2680 n 198.

${ }^{10}$ Voir Ansart, Marx et l'anarchisme, Paris, PUF, 1969, p. 121-123, ainsi que le jugement de François Perroux rapporté par P. Musso, op. cit., p. 196.

${ }^{11}$ Musso, op.cit., p. 199 ; voir encore $O C$ III, p. 2326 : avec Du système industriel, Saint-Simon fait « passer au premier plan les préoccupations politiques et pratiques ».

${ }^{12} O C$ III, p. 2482-83; encore une affirmation au futur ! cf. p. 2478 : « le besoin d'une doctrine philosophique proportionnée à l'état des lumières est aujourd'hui le besoin le plus grand du corps social, [..] celui qui est le moins susceptible d'ajournement ».]

${ }^{13}$ Pickering, op. cit., p. 186-191. Saint-Simon, estiment les éditeurs des $O C$, «bénéficie encore de l'aide, en passe de devenir magistrale, d'Auguste Comte, coauteur ou, à tout le moins, réviseur invisible, de la plupart, sinon de la totalité, des pièces détachées assemblées pour former Du système industriel » (OC IV, p. 3303) et
} 
nombreux passages du Système industriel ont un air de déjà-vu, de sorte qu'on ne peut échapper à la question : en 1822, Auguste Comte se réapproprie-t-il son bien, ou annexe-t-il celui de Saint-Simon ? Quelle qu'en soit l'importance, il n'est malheureusement pas possible d'aborder ici cette question.

Du système industriel et le programme de Saint-Simon. Ces préalables une fois posés, que trouve-t-on dans les écrits de Saint-Simon au moment où celui-ci s'apprête à publier le troisième cahier du Système industriel ? En dépit de la place considérable qu'ils occupent, on peut écarter certains thèmes, dans la mesure où ils n'interviendront pas dans ce qui suit. C'est le cas par exemple de la défense du point de vue industriel, mais aussi de la nécessité d'instaurer un nouveau pouvoir spirituel, de passer d'un système de gouvernement à un système d'administration, ou encore du parallèle entre la France et l'Angleterre.

Le programme que s'est fixé Saint-Simon apparaît alors composé de deux ou mieux de trois parties. Comme on vient de le voir, Saint Simon poursuit un but théorique, formuler la doctrine qui puisse servir de base au régime industriel; il poursuit aussi un but pratique, proposer des mesures qui doivent favoriser l'avènement d'un tel régime. Mais seule une analyse de la marche de la civilisation nous permet de savoir vers quoi nous nous dirigeons, de sorte que la première composante se subdivise, la partie proprement doctrinale reposant sur un travail historique préalable. Précisons le rapport entre ses trois éléments.

Au moins depuis 1814, le fil directeur est fourni par la volonté de terminer la révolution et de réorganiser la société européenne. De ce point de vue, la priorité des priorités est bien l'élaboration d'une doctrine nouvelle. C'est en effet une des convictions les plus profondes de Saint-Simon: «un système quelconque (et par conséquent un système politique) ne peut pas être remplacé par la critique qui l'a renversé. Il faut un système pour remplacer un système ${ }^{14}$. Nouveau, cela veut dire industriel, ce qui enveloppe un désaveu du système anglais : la Charte ne nous donne pas la doctrine dont nous avons besoin (OC III, p. 2545-2559), critique qui prépare celle du libéralisme. Nouveau, cela veut aussi dire positif : la politique doit prendre exemple sur les sciences et s'appuyer sur l'observation. Sur ce tronc viennent alors se greffer les deux autres parties.

Il convient en effet d'interroger tout d'abord le passé et d'étudier la marche de la civilisation. Si l'histoire est le bréviaire des rois (OC II, p. 1195 ; en 1819, Saint-Simon ajoutera « et des peuples » (OC III, p. 2146)), ce n'est pas tant qu'elle nous fournit des exemples de modèles à imiter ou d'erreur à éviter - ce qui était la conception traditionnelle - , mais parce qu'elle nous fournit les observations sur lesquelles doit désormais s'appuyer la politique. C'est donc le préalable indispensable à l'établissement d'une saine doctrine. A la différence des deux autres composantes du programme de Saint-Simon, l'importance de celle-ci risque facilement de passer inaperçu ${ }^{15}$. On sait que Comte a fait de Condorcet son principal précurseur ; mais Saint-Simon pourrait en dire autant. Le créateur de la mathématique sociale est chez lui une référence constante, et cela dès le début puisque, dès 1804, il déclarait: «j'entreprends de continuer l'ouvrage de Condorcet. - je présente un plan d'organisation » (OC I, p. 150; Lettres de deux philanthropes), affirmation qui met bien en évidence le lien étroit entre ces deux composantes du programme ${ }^{16}$. Il s'agit de déduire le futur du passé, ce qui s'accompagne d'une approche de la succession des temps dont Saint-Simon souligne l'originalité. L'ordre des temps n'est pas : passé, présent avenir, mais : passé, avenir, présent, celui-ci n'étant que «le point de réunion » des deux séries qui l'entourent (OC II, p. 1217). S'il faut «refaire Condorcet» (OC IV, p. 2958-59), c'est qu'il a mal découpé la succession des époques, et aussi qu'en homme du dix-huitième siècle, il n'a pas su rendre justice au moyen-âge.

Ce n'est qu'une fois en possession d'une doctrine fondée sur l'histoire, qu'il est possible de proposer des mesures concrètes adaptées à l'état actuel de la civilisation et de s'adresser aux différents acteurs de la vie politique : au Roi, pour lui proposer de promulguer des Ordonnances (OC III, p. 2575-78), aux industriels sur la loi des élections (OC III, p. 2300-2322). La logique voudrait que cette série de travaux soit exécutée dans l'ordre suivant : l'histoire (déterminer la marche de la civilisation), la doctrine (le nouveau but), les mesures à prendre pour atteindre ce but. S'il n'en est pas ainsi, si de fait la priorité est accordée aux mesures concrètes, objets des plus longs développements, c'est qu'il faut aussi tenir compte des demandes du public, tout particulièrement de celles des bailleurs de fonds, et pour cela répondre aux sollicitations de l'actualité et prendre parti dans la vie politique.

Gouhier (op.cit. p. 286-89; voir encore p. 265) avait présenté diverses hypothèses sur la nature de cette collaboration.

${ }^{14}$ OC III, p. 2115 (prospectus de L'Organisateur); cf encore OC III p. 2383 et François Dagognet, Trois philosophes revisités : Saint-Simon, Proudhon, Fourier, Hildesheim, Olms, 1997, p. 15.

${ }^{15}$ Dautry, op. cit., lui consacre une section ; voir aussi Musso, op. cit.

${ }^{16}$ Saint Simon fait même remonter l'articulation entre ces deux aspects à son séjour en Amérique, au moment où il renonce à la carrière militaire «Etudier la marche de l'esprit humain, pour travailler ensuite au perfectionnement de la civilisation, tel fut le but que je me proposai. Je m'y vouais dès lors sans partage » $(O C$ II, p. 1478). 
2. Comment Saint-Simon décrivait sa collaboration avec Comte.

Dans les quelque deux cents pages des Euvres complètes qui couvrent la période qui nous intéresse, le nom de Comte n'apparaît en tout et pour tout que deux fois; s'y ajoutent cinq autres références, plus ou moins explicites, mais toujours assez aisément identifiables. Ce premier ensemble de textes doit toutefois être complété par un second, où est précisée la nature du travail scientifique confié par Saint-Simon à son collaborateur.

Sept ou huit mentions. Commençons par la fin : en avril-mai 1824, dans le troisième cahier du Catéchisme, Saint-Simon présente en ces termes la seconde édition du Plan :

«Ce troisième cahier est de notre élève Auguste Comte. Nous lui avions confié, ainsi que nous l'avons annoncé dès notre première livraison, le soin d'exposer les généralités de notre système. C'est le commencement de son travail que nous allons mettre sous les yeux du lecteur » (OC IV, p. 2976)

Cette publication, comme il est rappelé dans le passage précédent, avait été annoncée quelques mois plus tôt, dans le second et dernier passage à citer le nom de Comte :

«Nous joindrons au troisième cahier du catéchisme un volume sur le système scientifique et sur le système d'éducation.

Ce travail, dont nous avons jeté les bases, et dont nous avons confié l'exécution à notre élève Auguste Comte, exposera le système industriel a priori, pendant que nous continuerons dans ce catéchisme son exposition a posteriori $\gg\left(O C \mathrm{IV}\right.$, p. 2900; $1^{\text {er }}$ Cahier du Catéchisme des industriels, déc. 1823)

Voilà pour les références explicites à Comte, mais il est possible d'en identifier d'autres. Ainsi, la quatrième de couverture du même premier cahier renvoie à l'annonce précédente. Sollicitant des souscriptions pour les six premiers cahiers du Catéchisme, Saint-Simon précise :

«Ces six cahiers, compris le travail qui a été annoncé à la page 46 [i. e. le passage cité ci-dessus] formeront deux volumes in octavo d'environ 25 feuilles chacun; ils seront publiés avant la fin de l'année $1824 »(O C$ IV, p. 2869).

Cette mention de deux volumes permet d'identifier une autre référence au Plan, dans le second cahier cette fois. En effet, renouvelant les appels à souscription, Saint-Simon résume les développements à venir et reprend en la modifiant, l'annonce précédente :

« Cet ouvrage formera deux volumes, dont un de catéchisme, et un autre contiendra l'exposé scientifique du système [c'est la tâche qui revient à Comte]. Ces deux volumes seront publiés en plusieurs livraisons, dans le cours de cette année $1824 »(O C$ IV, p. 2947).

Tout ceci se trouve dans le Catéchisme, i. e. dans les mois qui ont précédé immédiatement la seconde publication du Plan. Mais, même si le nom de Comte n'y apparaît pas, les déclarations les plus éclairantes sur la façon dont Saint-Simon concevait leur collaboration sont à chercher dans des textes antérieurs, à commencer par sa présentation de la première édition du Plan, dans la troisième partie du Système industriel, en mai 1822. S'adressant aux chefs des travaux de culture, de fabrication et de commerce, il déclare :

«Ce sont les savants qui doivent commencer les travaux qu'exige la réorganisation sociale.

Pour les déterminer à employer leurs forces et leurs talents dans cette direction, il était nécessaire que mon système leur fût présenté sous la forme scientifique.

Un de mes collaborateurs et amis s'est chargé de cette importante opération. Voici son travail, qui correspond au Discours préliminaire de l'Encyclopédie, par d'Alembert.

Je vous présenterai à la suite de cette pièce fondamentale les mesures que vous devez prendre pour activer les travaux des savants, relativement à la question qui nous occupe, sans vous mettre dans leur dépendance $»(O C \mathrm{IV}, \mathrm{p} .2773)$.

De là, comme pour la seconde publication, il est possible de remonter à des annonces antérieures, toutes deux de janvier 1822. Dans l'avant propos des Travaux philosophiques, scientifiques et poétiques ayant pour objet de faciliter la réorganisation de la société européenne, on lit :

« nous ferons paraître incessamment le travail scientifique et le travail poétique » (OC IV, p. 2761).

Peu avant, une brochure en prise avec l'actualité : Des Bourbons et des Stuarts s'achevait par

«Je publierai incessamment un travail plus étendu que ne l'est cette brochure. Il aura pour titre: $D u$

contrat social. [...] Mon prochain écrit sur le Contrat social éclaircira les obscurités qui pourront rester dans l'esprit du lecteur après qu'il aura lu celui-ci » (OC IV, p. 2750-2751).

De fait, trois mois plus tard, en avril, Saint-Simon publie Suite des travaux ayant pour objet de fonder le système industriel. Du contrat social, brochure dont les éditeurs nous disent (OC IV, p. 2756) : «Seules, les premières pages [..] sont de Saint-Simon. [...] La suite du texte [à savoir le Plan des travaux] est sans conteste d'Auguste Comte ». Il y a donc toute raison de penser que c'est bien l'opuscule de Comte qui est ainsi annoncé. A ces sept passages, il y a sans doute lieu d'en ajouter un dernier. Parmi les trois manuscrits de mars 1823 restés inédits jusqu'en 1970 figure un «Prospectus d'un ouvrage ayant pour objet la production du système d'organisation sociale qui correspond à l'état présent de la civilisation en Europe ». De l'ouvrage ainsi annoncé, les éditeurs nous disent: «le sujet et la démarche historique annoncée inspireront sans doute à 
l'Histoire générale de la civilisation en Europe, le cours d'histoire moderne que Guizot professera et publiera en 1828 » (OC IV, p. 3232). Mais on conçoit mal comment un texte resté enfoui dans les papiers de l'auteur aurait pu exercer quelque influence que ce soit et, compte tenu de ce que nous savons par ailleurs, la date, $1^{\text {er }}$ mars 1823, invite à penser que ce prospectus a quelque rapport avec Comte. Comme on le verra plus tard, en effet, Saint-Simon était alors dans une situation critique. Il attendait avec la plus grand impatience la suite du plan de 1822 et c'est quelques jours après avoir appris que le texte n'était pas prêt qu'il a tenté de mettre fin à ses jours.

Il y a donc de bonnes raisons de supposer que ce qu'annonce le prospectus de mai 1823 n'est autre que ce que Comte peu après s'engagera par contrat à fournir. Et si le prospectus reste dans les cartons, c'est précisément qu'il devient sans objet après le refus de Comte. Contre cette hypothèse, on peut faire valoir que les développements historiques qui occupent le gros du texte n'ont pas grand-chose à voir avec les vues historiques de Comte ; mais c'est oublier que le contrat prévoira une « esquisse d'un tableau historique du progrès de la civilisation ». On peut supposer que, faute d'éléments, et pressé par les circonstances, Saint-Simon puise dans son propre fond, dans l'idée que cela ne prête pas trop à conséquence. Dans l'ensemble donc, et compte tenu de ce que nous savons par ailleurs de Saint-Simon au début mars 1823, il est raisonnable de considérer ce prospectus comme annonçant le texte que Saint-Simon espérait recevoir prochainement de celui qui était encore son secrétaire.

Un travail scientifique: science, histoire et philosophie. De ce qui précède, il ressort qu'il y avait pour SaintSimon plusieurs façons de décrire sa collaboration avec son secrétaire. Tantôt il parle d'exposé a priori, se réservant l'exposé a posteriori ; tantôt il confie à Comte le soin d'exposer les généralités de son système (OC IV, p. 2976 ; entendez : le système industriel). De fait, les brouillons de Comte de 1821, où on voit apparaître pour la première fois les premières lignes du Plan, portent pour titre «Du système industriel, $2^{e}$ partie $»^{17}$. Toutefois, la description la plus fréquente assigne, à celui qui est d'abord désigné comme collaborateur et ami puis simplement comme élève, la partie scientifique du travail. Mais que faut il entendre au juste par là ? La réponse n'allant pas de soi, il convient d'élargir le corpus et de considérer deux autres textes précisant les rapports de la science à l'histoire, et à la philosophie.

Que le travail scientifique comporte une dimension historique, c'est ce que nous apprend le contrat passé entre Saint-Simon et Comte, reproduit en appendice, par lequel ce dernier s'engageait à remettre « un volume qui se compose pour la première partie du plan des travaux scientifiques nécessaires pour réorganiser la société et pour la second partie de l'esquisse d'un tableau historique du progrès de la civilisation $»^{18}$. Si Comte ne fut jamais en mesure de fournir la seconde partie promise, sa présence ne doit pas nous surprendre, l'histoire étant considérée par les deux hommes comme un science ${ }^{19}$. C'est parce qu'il porte sur les généralités du système industriel que le même travail peut également être considéré comme philosophique. Depuis longtemps en effet, pour SaintSimon, la philosophie est une science, la science générale ${ }^{20}$. C'est à ce titre que les philosophes sont seuls en mesure d'élaborer la doctrine qui doit servir de base au régime industriel, comme le constate Saint-Simon en décembre 1820, au moment où il résume et conclut la première partie du Système industriel :

«Les savants ont bien, et les éléments du travail théorique nécessaire pour la formation de la doctrine industrielle, et les dispositions intellectuelles indispensables pour cela. Mais il leur manque l'idée générale de ce travail, sans laquelle néanmoins il ne pourrait être mis en activité, puisqu'il faut qu'un noyau de doctrine serve de lien aux éléments complets, mais isolés, que les savants possèdent ; pour que la combinaison de leurs capacités individuelles puissent avoir lieu. C'est aux philosophes positifs, c'est-àdire aux hommes occupés à observer et à coordonner les généralités positives, qu'il appartient de remplir cette importante fonction » (OC III, p. 2484).

La plupart des savants sont prisonniers de leur spécialisation, et le travail scientifique attendu de Comte relève donc également de la philosophie, entendue comme science générale.

\footnotetext{
${ }^{17}$ Voir Gouhier, op. cit., p. 287 n. 22 et Pickering, op. cit., p. 192. On notera que l'expression n'appartient pas vraiment au vocabulaire de Comte, qui l'emprunte à Saint-Simon.

${ }^{18}$ A Tabarié, 5 avril 1824, CG I, p. p. 77 : «il avait été convenu, comme vous le savez, que mon volume serait composé de deux parties, celle faite déjà, et une autre relative à l'histoire de la civilisation. Celle-ci d'abord devait précéder, mais j'ai reconnu en travaillant qu'elle devait suivre, et comme ce changement a occasionné un nouveau retard dans son exécution [...] nous convînmes que la partie exécutée depuis deux ans paraîtrait d'abord seule ».

19 «Son ouvrage [de Condorcet] que nous avons refait, et que nous publierons incessamment» (OC IV, p. 2958-59) : On peut voir là une nouvelle allusion au texte promis par Comte. Le titre figurant dans le contrat étant calqué sur celui de Condorcet et on voit mal publier simultanément deux textes sur le même sujet.

${ }^{20}$ « La science générale ou philosophique a pour faits élémentaires les faits généraux des sciences particulières ou, si l'on veut, les sciences particulières sont les éléments de la science générale » (OC II, p. 1081, cf. p. 1075 ; Mémoire sur la science de l'homme, 1813).
} 
3 Les circonstances de la rupture et leur enseignement.

Si l'on veut maintenant comprendre comment cette collaboration prit fin, il convient de compléter les indications fournies dans les écrits de Saint-Simon en les rapportant à ce que nous savons de sa vie à cette époque, d'après les témoignages qui nous sont parvenus sur cette période.

Les circonstances. Commençons par rappeler quelques faits. Comme on l'a vu, la publicité faite à la parabole de Saint-Simon avait permis à celui-ci de lancer une nouvelle publication, Du système industriel. Mais les ressources n'étaient pas inépuisables. Quelques mois après la première édition du Plan, en juillet 1822, SaintSimon cesse toute publication. Il est en effet pris dans un cercle vicieux : «plus d'argent, plus de parution; plus de parution, plus d'argent» $(O C \mathrm{IV}$, p. 3305). Son principal commanditaire, Ternaux, est prêt à le sortir d'affaire, mais à une condition: "l'achèvement de l'œuvre dont la rédaction avait été confiée à monsieur Comte $»^{21}$. Le témoignage de Pierre Leroux rapporté par Joseph Bertrand va dans le même sens : Saint-Simon attendait avec impatience le texte que son secrétaire devait lui remettre, «les annonces étaient répandues à profusion, lorsque Comte fit savoir qu'il n'était pas prêt $»^{22}$. C'est alors que Saint-Simon, après avoir écrit à Ternaux, se tira une balle dans la tête. Comte accourut au chevet de son patron, Saint-Simon survécut, et put reprendre quelques mois plus tard ses publications, grâce notamment à l'aide d'Olinde Rodrigues, dont il fit la connaissance en mai 1823.

Les enseignements. Le premier enseignement de cet épisode, tout lecteur de l'opuscule fondamental peut, aujourd'hui encore, en faire par lui-même l'expérience : Il s'agit d'une œuvre comme on a peu l'occasion d'en lire. Comte le sait, et Saint-Simon aussi qui, en 1824, présente l'œuvre comme «le meilleur écrit qui ait jamais été publié sur la politique générale $»^{23}$. Tirée à un tout petit nombre d'exemplaires, la première édition, si elle connut une diffusion très limitée, ne manqua pas de produire une forte impression sur ses lecteurs. Comte confia un jour à Pierre Laffitte « qu'après la semi publication de l'opuscule fondamental de 1822, il se trouvait chez Saint-Simon lorsque M. Ternaux vint voir celui-ci et lui parla avec grande admiration du travail d'Auguste Comte. Montrant celui-ci, il dit: C'est Monsieur qui a rédigé ce travail remarquable. Saint-Simon répondit : Rédigé! Oh! Mieux que cela $»^{24}$. Que l'anecdote soit vraie ou fausse, elle témoigne de l'effet produit par l'ouvrage. Et ce qui vaut de Ternaux vaut encore de Blainville, de Humboldt, de Guizot ou un peu plus tard de Mill, lorsque en 1828 Gustave d'Eichthal, qui se donnait encore pour un disciple de Comte, lui fit lire l'opuscule fondamental ${ }^{25}$.

Le deuxième enseignement concerne plus directement les rapports de Comte et de Saint-Simon. S'il est vrai que, en 1824, ce dernier présente le premier comme son élève, il est également assez clair qu'il avait reconnu en lui un pair, et même, dans certains domaines, un supérieur. Ainsi, en mars 1822, au moment de la première

${ }^{21}$ G.Hubbard, Saint-Simon, sa vie et ses travaux, Paris, Guillaumin, 1857, p. 92-93, cité dans Gouhier, op.cit., p. 361, qui renvoie également à un projet de lettre de G. d'Eichthal.

${ }_{22}$ Olivier Pétré-Grenouilleau, (Saint-Simon, l'Utopie ou la raison en actes, Paris, Payot, 2001), semble ignorer ces deux témoignages. Dans le récit qu'il donne de cet épisode, Comte n'apparait qu'après la tentative de suicide : «pensant son heure arrivée, Claude-Henri passa ensuite la nuit à dicter des notes à Auguste Comte » (p. 370).

${ }^{23}$ OC IV, p. 2977 ; cf. encore les commentaires des éditeurs de 1875, dans Claude-Henri de Saint-Simon, Euvres complètes, Paris, Editions Anthropos, 1966, t. IV, p. ii-iii.

${ }^{24}$ Gouhier, op. cit. p. 359, qui renvoie à Revue Occidentale, 1893, t. 2, p ; 320-321 et 1895, t. 1, p. 6-7..

${ }^{25}$ Pour Humboldt et Guizot, voir la lettre à d'Eichthal du $1^{\text {er }}$ mai 1824 (CG I, p. 83) ; pour Mill, voir M. Bourdeau : "Comme un rayon de lumière à travers les ténèbres, la correspondance de Mill et de Gustave d'Eichthal (1829-1871)», Cahiers philosophiques, 148, 2017, p. 105-126 et la lettre du 29 octobre 1829: « le maître a été surpassé par l'élève » (cité dans Pickering, op. cit., p. 246-47, qui signale que la lettre ne se trouve pas dans l'édition de la correspondance dans les Collected Works publiées aux presses de l'université de Toronto).

Voir encore la quinzième séance de l'Exposition de la doctrine de Saint-Simon (Digression sur l'ouvrage intitulé Troisième cahier du catéchisme des industriels, par Auguste Comte, élève de Saint-Simon) : « Le travail de M. A. Comte a servi à plusieurs d'entre nous d'introduction à la doctrine de Saint-Simon ; [...] Si on le considère du point de vue où l'auteur s'est placé [...] aucun essai produit par la capacité scientifique pure ne lui est comparable » (3 ${ }^{\mathrm{e}}$ édition, Paris, aux bureaux de l'Organisateur, 1831, p. 373-374). Hayek, peu suspect pourtant de sympathie pour celui où il voyait le prototype du constructiviste, reconnaissait dans le Plan de 1822 « one of the most pregnant tracts of the 19th century » (Collected Works, Chicago, The University of Chicago Press, vol. 13 (Studies on the Abuse \& Decline of Reason, (Br. Caldwell ed.), p. 293 ; cf. encore « It is the most significant single tract of the whole litterature with which we are here concerned » (p. 212)). 
publication, il n'hésite pas à présenter le Plan comme « un travail qui correspond au Discours préliminaire de l'encyclopédie, par d'Alembert » (OC IV, p. 2773).

Pour mesurer toute la portée de cette déclaration, il convient de remonter aux écrits sur la science de 1807-1808 (Lettres au bureau des longitudes, Introduction aux travaux scientifiques du XIX siècle). D'Alembert, et le Discours préliminaire de l'Encyclopédie y occupent en effet une place de premier plan. Ce à quoi visent les projets d'encyclopédie élaborés à l'époque par Saint-Simon, c'est à refaire d'Alembert, sur le travail de qui un jugement très critique est porté : «Cette encyclopédie a été faite d'après un mauvais plan, dans un mauvais esprit, elle a été mal exécutée » $(O C \mathrm{I}$, p. 650$)$; «D'Alembert est entré dans la carrière la tête basse et les jambes tremblantes. Quel pitoyable début » $(O C$ I, p. 664) ; «Les encyclopédistes, dis-je, ont travaillé bien plus efficacement à la démolition de l'ancien système qu'à la construction du nouveau » (OC I, p. 299). Il est en particulier indispensable d'actualiser le Discours Préliminaire (OC I, p. 215, 441-442, 530-538, 657-82). Ce n'était pas à Augustin Thierry qu'on pouvait demander un tel travail mais, après 1817, la situation change. Saint-Simon étant maintenant tout occupé par la politique, c'est à Comte qu'est confié le soin de mener à bien le programme élaboré quelque dix ans plus tôt. Le Plan de 1822 n'est donc rien moins que la réalisation d'un des objectifs fondamentaux que s'était fixé Saint-Simon. Le Discours préliminaire de l'Encyclopédie est refait, et le programme des Lumières est enfin rempli, en ce sens que nous disposons d'une vision unifiée des sciences, conçue cette fois d'un point de vue constructif, de sorte que les travaux scientifiques pourront désormais contribuer à réorganiser la société. Une fois la part faite à ce qui, dans ce parallèle établi avec d'Alembert, relève des soucis promotionnels d'un éditeur, c'est bien la conscience d'être en présence d'un esprit supérieur qui s'exprime.

Cette conscience, on peut en trouver une expression plus ancienne dans un passage de la sixième lettre de la première partie du Système industriel, Résumé des lettres sur les Bourbons (décembre 1820), sur lequel Mary Pickering a justement attiré l'attention. Saint Simon, qui vient d'affirmer que tous ses travaux ultérieurs auront désormais pour objet l'établissement d'une doctrine qui puisse servir de base au régime industriel, et que ce travail, quoique scientifique, devait être confié aux philosophes positifs, seuls à même d'observer et de coordonner les généralités positives, poursuit :

«Aucun philosophe ne se présentant pour obéir à cette grand mission que l'état de la civilisation met réellement à l'ordre du jour, j'ai osé m'en charger. Je serai heureux si mon travail peut déterminer à s'en occuper un philosophe positif plus habile, ou si, bientôt, je puis avoir assez avancé l'entreprise pour pouvoir la remettre entre les mains des savants, ce qui est l'objet de tous mes vœux » (OC III, p. 2484). Pour Mary Pickering, «Saint-Simon a manifestement Comte en tête ${ }^{26}$. Le nom de ce dernier n'étant pas mentionné, il est difficile d'être catégorique, mais, si Saint-Simon pense à quelqu'un (et s'il ne pensait à personne, pourquoi évoquerait-il une telle possibilité ?), on a toute raison d'estimer, comme elle, que ce " philosophe positif plus habile» n'est autre que Comte. C'est d'ailleurs peu après, en avril 1821, que celui-ci commença la rédaction de ce qui allait devenir l'opuscule fondamental.

Tout ceci s'accorde bien avec ce que nous savons par ailleurs. C'est à Comte que revient, dans le programme de Saint-Simon, la partie scientifique, dont on a vu qu'elle inclut la philosophie et l'histoire. Comme par un accord tacite, s'est instaurée entre les deux hommes une sorte de division du travail. Conformément à une évolution déjà signalée, Saint-Simon délaisse le travail encyclopédique pour s'occuper en priorité des questions politiques, et plus précisément encore, des mesures pratiques, Comte se trouvant ainsi chargé du travail proprement théorique. C'est à lui qu'est confié la partie la plus difficile et la plus importante, aux dires de SaintSimon lui-même, «celle de concevoir, d'une manière claire, le système industriel » (OC IV, p. 2899). C'est d'ailleurs ce que Saint-Simon lui reprochera en 1824 : «notre élève n'a traité que la partie scientifique de notre système, mais [..] il n'a point exposé sa partie sentimentale et religieuse $»^{27}$.

Mais les relations entre les deux hommes n'étaient pas qu'intellectuelles. Elles comportaient aussi une dimension institutionnelle et là rien n'avait changé depuis 1817. Le supérieur était toujours Saint-Simon. Chacun en était conscient et le besoin qu'éprouvait Comte de s'émanciper d'une tutelle qui lui pesait de plus en plus a joué un rôle décisif dans la rupture. Cette indépendance a d'ailleurs un coût et, privé de l'appui de son mentor, Comte savait qu'il se retrouverait dans une situation matérielle difficile. La situation illustre un phénomène bien mis en valeur dans la loi positive de classement : la relation de subordination fonctionne dans les deux sens et le supérieur dépend aussi de l'inférieur. Comme le remarquait Comte, Saint Simon «a, en définitive, beaucoup plus besoin de moi, que moi de lui $»^{28}$.

${ }^{26}$ Pickering, op. cit., p. 192.

${ }^{27} O C$ IV, p. 2976; ce reproche témoigne de l'évolution de Saint-Simon à cette époque : avec Le nouveau christianisme, il prenait ses distances à l'égard de la science. On notera que Comte a suivi une évolution similaire.

${ }^{28}$ Lettre à Tabarié du 5 avril 1824, $C G$ I p. 78. 
La rupture une fois consommée, Comte tentera pendant deux ans encore de mener à bien l'ouvrage laissé inachevé. Interrompue depuis plusieurs années (du moins pour ce qui nous en est parvenu), la correspondance reprend, et abondante, juste au moment où tout se gâte : «le travail s'imprime en ce moment » écrit Comte à Tabarié le 5 avril (CG I, p. 77). Grâce à elle, nous pouvons suivre les multiples efforts entrepris, jusqu'à la lettre tout à fait capitale adressée à Blainville le 26 février 1826, où il se rend à l'évidence et dresse un constat d'échec. Le Cours de philosophie positive qu'il s'était engagé à donner du $1^{\mathrm{er}}$ mars 1826 au $1^{\mathrm{er}}$ mars 1827 l'avait en effet conduit «à concevoir une refonte totale» de l'ouvrage en cours : «je suis donc parvenu aujourd'hui à voir très distinctement que cet ouvrage (même en le supposant complété par ma deuxième partie) ne remplissait pas complètement les conditions d'un véritable système ${ }^{29}$. Comte expose alors un plan de refonte, - qu'il ne suivra pas —, qui s'ouvrait par les deux articles qu'il venait de publier dans Le Producteur, et qui devait «montrer la formation de [1]a physique sociale comme étant, soit sous le rapport de la doctrine, soit même sous celui de l'organisation, un commencement direct de reconstruction » (CG I, p. 188). Si, comme l'indique l'avertissement de 1824 reproduit ici en appendice, ses efforts avaient jusque là tendu à " présent[er] un premier coup d'œil scientifique sur les lois qui ont présidé à la marche de la civilisation », on comprend qu'il ait reculé devant la tâche. Il ne s'agit en effet de rien moins que la dynamique sociale, c'est-à-dire de ce qui représente la plus grande partie des leçons de sociologie du Cours, et la sociologie ne pouvait être constituée qu'une fois accomplie la revue des sciences qui occupe les trois premiers volumes du Cours. Retracer le chemin suivi par Comte de 1824 à 1826 n'entre pas dans le cadre fixé à cette étude ${ }^{30}$ et il suffira de retenir : a) qu'un temps, Comte a, semble-t-il, espéré pouvoir se réconcilier avec Saint-Simon, dont les réseaux lui étaient fort utiles $^{31}$; b) que les deux articles du Producteur qui suivirent l'opuscule fondamental entretiennent avec celui-ci un double rapport : d'une part, ils en constituent comme la continuation, d'autre part, c'est à leur occasion que leur auteur s'est rendu compte de son incapacité à réaliser le programme qu'il s'était initialement fixé.

Il est clair que l'initiative de la rupture de 1824 revient à Comte ; clair également que la version que celui-ci en a donnée aussitôt après à ses correspondants ne tient pas. Il est faux que les difficultés rencontrées dans la republication du Plan de 1822 soient imputables à Saint-Simon, qui, par crainte que l'œuvre de son collaborateur ne lui porte ombrage, aurait tout fait pour empêcher la diffusion de l'opuscule fondamental ${ }^{32}$. Il est clair enfin qu'il est impossible d'accepter la description que Comte par la suite a constamment donné de ses rapports avec Saint-Simon et il serait utile de dresser un inventaire circonstancié de ce qu'il doit à celui dont il fut pendant sept ans le secrétaire.

Si personne ne songe donc à nier l'influence de Saint-Simon sur Comte, on passe d'ordinaire sous silence l'influence de celui-ci sur celui-là, alors pourtant que la question est tout aussi légitime, l'étroite collaboration menant assez rapidement à une influence réciproque. Dans la mesure où personne ne conteste à Comte la pleine paternité de l'opuscule de 1822, le problème ne se pose pas à son sujet. Il n'en va pas de même pour les textes des années antérieures, au moment où la collaboration entre les deux hommes était très étroite, et les travaux $\mathrm{d}^{\prime}$ Irène Manfredini ${ }^{33}$ donnent une assez bonne idée de la difficulté qu'il y a à démêler ce qui revient à l'un et à l'autre. Sans en attendre des éclaircissements définitifs, des études stylométriques, comme celles qui dès la fin du XIXe siècle, ont permis de débarrasser le corpus platonicien de textes apocryphes, devraient nous aider à avancer un peu. Pour une meilleure connaissance de ces textes qui appartiennent à une époque d'étroite collaboration entre les deux hommes, il serait également souhaitable que s'instaure une collaboration entre ceux qui s'intéressent à l'un et ceux qui s'intéressent à l'autre.

\section{Appendice}

Les deux textes qui suivent ne sont pas inédits mais ils apportent, sur la période étudiée, de précieuses informations et sont difficiles d'accès. En particulier, ils ne figurent pas dans les Ecrits de jeunesse publiés en 1970 par Paulo Carneiro et Pierre Arnaud. C'est pourquoi il a paru utile de les reproduire ici.

\section{Contrat par où Comte s'engage à fournir à Saint-Simon la suite du Plan de 1822}

\footnotetext{
${ }^{29}$ CG I, p. 187. Pickering, op. cit., p. 329 signale que le texte de la Correspondance générale est fautif et qu'il faut lui préférer celui qui figure dans Correspondance inédite d'Auguste Comte, Paris, au Siège de la Société Positiviste, 1903, t. 1, p. 17-26. Sur cette lettre capitale, voir aussi Gouhier, op. cit., p. 312. On peut considérer que cette lettre met un terme à la période de ce qu'on appelle les écrits de jeunesse, le dernier d'entre eux, l'Examen du traité de Broussais sur l'irritation, ayant été composé après la crise cérébrale survenue quelques jours plus tard, et relevant d'une toute autre orientation.

${ }^{30}$ Voir Pickering, op. cit., p. 328-357, et Gouhier, op. cit., p. 300-320.

${ }^{31}$ Voir la lettre à Tabarié du 5 avril 1824, CG I p. 77. Mary, p ??

${ }^{32}$ Voir Gouhier, op. cit., p. 369, 371.

${ }^{33}$ Irène Manfredini, Saint-Simon. Les manuscrits de L'industrie ; Florence, Leo Olschki, 1999.
} 
Manuscrit d'une page recto-verso, conservé dans les archives de la Maison Auguste Comte (cote : D.COM.64), ni signé ni daté mais postérieur à mai 1823. Il a été publié pour la première fois dans E. Sémerie, La loi des trois états, réponse à Monsieur Renouvier, Paris, E. Leroux, 1875, p. 19-20, et reproduit par Teixeira Mendes dans Auguste Comte, évolution originale, Rio, au siège central de l'Eglise positiviste du Brésil, 1913, p. lxxxiilxxxiii. L'orthographe et la ponctuation ont été modernisées.

« Entre nous soussignés il a été convenu ce qui suit

Moi Auguste Comte vends à Henry St-Simon aux conditions suivantes un volume qui se compose pour la première partie du plan des travaux scientifiques nécessaires pour réorganiser la société et pour la second partie de l'esquisse d'un tableau historique du progrès de la civilisation. Le volume sera imprimé aux frais de Monsieur St-Simon qui aura indéfiniment le droit de le faire réimprimer.

Mr. St Simon me remettra cent exemplaires de la première édition de ce volume.

A partir du premier avril 1825 j'aurai le droit ainsi que Mr. St-Simon de faire faire autant d'éditions que je voudrai de ce travail.

Si je fais des additions ou corrections à ce travail Monsieur St-Simon aura indéfiniment droit de le faire imprimer avec ses corrections ou additions.

Monsieur St-Simon me payera la somme de deux mille quatre cent francs en douze payements de deux cents francs chacun. Le premier payement aura lieu aussitôt que le volume sera imprimé et les onze autres payements auront lieu de mois en mois.

Moi St-Simon accepte les dites conditions.

Il a été convenu en outre que s'il s'élevait quelques discussions entre nous relativement à ce traité nous choisissons d'avance Monsieur Olinde Rodrigue pour arbitre et que nous admettrons le jugement qu'il prononcera comme jugement définitif

Fait double »

\section{L'avertissement de 1824}

Le texte se trouve en tête de la seconde édition du Plan, qui porte pour titre : Système de politique positive; d'où au sixième alinea, le rappel de la décision de substituer un nouveau titre à celui de Système industriel, longtemps utilisé par Saint-Simon. Il est reproduit dans E. Littré, Auguste Comte et la philosophie positive, Paris, Hachette, 1863, p. 20-22 ; et dans Claude-Henri de Saint-Simon, Euvres complètes, Paris, Editions Anthropos, 1966, t. IV, p. 6-9.

De cet avertissement, on retient d'ordinaire ${ }^{34}$ l'acte d'allégeance à Saint-Simon, qui est à prendre cum grano salis quand on connaît les circonstances dans lesquelles il a été écrit. Ce qu'on peut en apprendre sur les projets de Comte à cette date (programme que Comte se fixait alors est tout aussi intéressant. Ce que Comte s'engageait à publier n'est plus que la première d'une série de trois travaux correspondants, peut-on penser, à ce que 1822 annonçait déjà (p. 271) : un traité d'éducation et un traité de l'action de l'homme sur la nature ; ouvrages que Comte continuera à promettre à la fin du Cours et dans le Système de politique positive. On comparera également la description donnée de la première série à celle qui figure dans l'opuscule fondamental (p. 288), toujours composée de deux parties. Enfin, ce qui est dit de la deuxième partie témoigne du chemin accompli depuis 1823. Il ne s'agit plus seulement de refaire Condorcet, même si cela doit encore s'achever par un tableau de l'avenir. Ce qui reste à écrire se rapporte à l'exécution du Plan, relève de la méthode, et s'il y est encore question de la marche générale de la civilisation, c'est en tant qu'elle offre « un premier aperçu du système social » vers lequel nous nous dirigeons.

« Cet ouvrage se composera d'un nombre indéterminé de volumes formant une suite d'écrits distincts, mais liés entre eux, qui tous auront pour but direct, soit d'établir que la politique doit aujourd'hui s'élever au rang des sciences d'observation, soit d'appliquer ce principe fondamental à la réorganisation spirituelle de la société.

Les deux premiers volumes, qui peuvent être regardés comme une sorte de prospectus philosophique de l'ensemble de l'ouvrage, contiendront à la fois l'exposition du plan des travaux scientifiques sur la politique, divisés en trois grandes séries, et une première tentative pour exécuter le plan.

Le premier volume est, en conséquence, composé de deux parties : l'une est relative au plan de la première série de travaux ; l'autre, qui sera publiée peu de temps après, se rapporte à son exécution.

Le but de la première partie est proprement d'établir, d'une part, l'esprit qui doit régner dans la politique, considérée comme une science positive; et, d'autre part, de démontrer la nécessité et la possibilité d'un tel changement. L'objet de la seconde est d'ébaucher le travail qui doit imprimer ce caractère à la politique, en présentant un premier coup d'œil scientifique sur les lois qui ont présidé à la marche générale de la civilisation, et, par suite, un premier aperçu du système social que le développement naturel de l'esprit humain doit rendre aujourd'hui dominant. En un mot, la première partie traite de la méthode, et la seconde de son application.

${ }^{34}$ Voir Gouhier, op. cit., p. 373-375, et $O C$ IV, p. 3242 n. 115. 
La même division sera observée dans le volume suivant, relativement aux deux autres séries de travaux.

Afin de caractériser avec toute la précision convenable l'esprit de cet ouvrage, quoiqu'étant, j'aime à le déclarer, l'élève de M. de Saint-Simon, j'ai été conduit à adopter un titre général distinct de celui des travaux de mon maître. Mais cette distinction n'influe point sur le but identique, ces deux sortes d'écrits, qui doivent être envisagés comme ne formant qu'un seul corps de doctrine, tendant, par deux voies différentes, à l'établissement du même système politique.

J'ai adopté complètement cette idée philosophique émise par M. Saint-Simon, que la réorganisation actuelle de la société doit donner lieu à deux ordres de travaux spirituels, de caractère opposé, mais d'égale importance. Les uns, qui exigent l'emploi de la capacité scientifique, ont pour objet la refonte des doctrines générales ; les autres, qui doivent mettre en jeu la capacité littéraire et celle des beaux-arts, consistent dans le renouvellement des sentiments sociaux.

La carrière de M. Saint-Simon a été employée à découvrir les principales conceptions nécessaires pour permettre de cultiver efficacement ces deux branches de la grande opération philosophique réservée au dixneuvième siècle. Ayant médité depuis longtemps les idées mères de M. Saint-Simon, je me suis exclusivement attaché à systématiser, à développer et à perfectionner la partie des aperçus de ce philosophe qui se rapporte à la direction scientifique. Ce travail a eu pour résultat la formation du système de politique positive que je commence aujourd'hui à soumettre au jugement des penseurs.

J'ai cru devoir rendre publique la déclaration précédente, afin que, si mes travaux paraissent mériter quelque approbation, elle remonte au fondateur de l'école philosophique dont je m'honore de faire partie.

Il est sans doute superflu de justifier ici de la loyauté de mes intentions politiques, et d'entreprendre de prouver l'utilité des vues que j'expose. Le public et les hommes d'Etat jugeront l'un et l'autre point à la lecture de cet ouvrage ; c'est à eux qu'il appartient de décider, après un mûr examen, si ces idées tendent à jeter dans la société de nouveaux éléments de trouble, ou à seconder, par des moyens spéciaux et dont le concours est indispensable, les efforts des gouvernements pour rétablir l'ordre en Europe. » 